\title{
Kurt-Victor Selge \\ Die Überlieferung der Werke Joachims von Fiore im 14./15. Jahrhundert
}

Datierung undatierter Handschriften ist ein Geschäft mit Fehlerquellen. Denn das Alter des Scriptors, der seine littera vielleicht zwanzig, vielleicht fünfzig Jahre lang nicht wesentlich veränderte, ist unbekannt. „Scriptor sum, talis monstrat me littera qualis“, schrieb Ende des 12. Jahrhunderts ein anonymer Scriptor am Ende seiner Arbeit ${ }^{1}$. Ist die littera chronologisch annähernd eingeordnet, was auch nur auf den Raum einiger Jahrzehnte möglich ist, bleibt das Alter der Handschrift, sofern nicht weitere Indizien vorliegen, nur im Zeitraum eines halben Jahrhunderts bestimmbar.

Ebenso fehlerträchtig ist die Lokalisierung der Handschrift, wenn nicht - bei Papierhandschriften - das Wasserzeichen des Papierproduzenten einen unsicheren Hinweis gibt. Denn der französische Zisterzienser in einem italienischen Kloster, der oft versetzte Bettelmönch - ihre einmal erworbene littera brachten sie mit. Und das Wasserzeichen für sich allein sagt nur, wann und wo das Papier produziert, nicht aber, wohin es verkauft wurde und wie lange es vor der Beschriftung lagerte.

Daß Joachims Werke, die echten wie die pseudonymen, bis ins frühe 16. Jahrhundert abgeschrieben wurden, bis sie in Venedig - von 1516 bis 1527 - gedruckt wurden $^{2}$, zeigt ein Blick auf die Überlieferung, ebenso wie es sich aus der kleinen Joachim-Renaissance im Umkreis des Augustinereremitengenerals Aegidius von Viterbo (1469-1532), des Freundes von Marsilio Ficino und Johannes Reuchlin, ergibt, aus der die venezianischen Drucke mit hervorgingen ${ }^{3}$. Aber die Altersangaben in der Hand-

1 Kurt-Victor Selge, Die ersten Waldenser. Mit Edition des Liber antiheresis des Durandus von Osca (Arbeiten zur Kirchengeschichte 37 I/II, Berlin 1967) II 257. Der Scriptorvers geht noch weiter: „Scribere qui nescit, nullum putat esse laborem. / Tres digiti scribunt, vix omnia membra quiescunt. / Laus tibi sit, christe, quoniam cessat labor iste."

${ }_{2}^{2}$ Super Hieremiam (Venedig 1516 und 1525); Super Esaiam (Venedig 1517); Concordia novi ac veteris testamenti (Venedig 1519); Expositio in Apocalypsim und Psalterium decem cordarum (Venedig 1527).

${ }_{3}$ Marjorie Reeves, The Influence of Prophecy in the Later Middle Ages (Oxford 1969) 268 gibt aus der Widmungsvorrede des Apokalypsenkommentars an Aegidius von Viterbo (1527) wieder, daß der Editor seit 1516, fr. Silvester Meucci, diesem die ersten Drucke vorgelegt hat und von ihm zur Fortsetzung dieser Tătigkeit, insbesondere zur Edition des Apokalypsenkommentars, aufgefordert worden ist. Über die Beziehungen Aegidius' z. B. zu Marsilio Ficino vgl. ebd. 433. Vgl. ferner Bernard McGinn, Circoli gioachimiti veneziani (1450-1530), in: Cristianesimo nella Storia 7 (1986) 19-39 (auf S. 32), und demnächst M. Reeves, Il Cardinale Egidio da Viterbo e l'Abate Gioacchino, in: Continuità e diversità nel profetismo gioachimita tra '400 e '500 (Atti del 
schriftenliste von Marjorie Reeves ${ }^{4}$ - die nicht vollstāndig ist - sind vage und bestätigen sich nicht durchweg. Einen krassen Fall stellt die schöne großformatige Handschrift des Werkes der „Concordia Novi ac Veteris Testamenti“ aus Padua dar (Bibl. Antoniana 328). Da sagt Frau Reeves: „1. Hälfte 13.Jh.“, weil die littera auf den ersten Blick ähnlich aussieht wie die der anderen Paduaner Miscellaneahandschrift (an der mindestens zehn Scriptoren gearbeitet haben), die in der Tat in diese Zeit gehört. Aber näheres Zusehen zeigt: der Schenkungsvermerk, nach dem der Petrarca nahestehende Bischof Ildebrandinus Conti (gest. November 1353) den Codex zu seinen Lebzeiten dem Konvent des $\mathrm{Hl}$. Antonius vermachte, ist vom Scriptor des ganzen Codex angefertigt worden, und zwar fand die Schenkung am 26. September 1352 statt. Der Codex dürfte also wenig älter und vom Bischof für seinen eigenen Gebrauch in Auftrag gegeben worden $\operatorname{sein}^{5}$.

Über die Motive, aus denen Bischof Ildebrandinus Wert darauf legte, diesen Codex anfertigen zu lassen, ist damit nichts gesagt. Und was mag später den Paduaner Bürger, Herrn Jacob de S. Cruce, an ihm interessiert haben, unter dessen Büchern er bei seinem Tode von dem Rechtsgelehrten Herm Paganinus a Sala aufgefunden wurde und der ihn dann später seinerseits dem "frater" Bartholomaeus de S. Georgio anvertraute? Durch ihn ist er dann wohl an den Konvent des Santo zurückgelangt ${ }^{6}$.

\section{Fortsetzung Fußnote von Seite 49}

$3^{\circ}$ Congresso Internazionale di Studi gioachimiti, S. Giovanni in Fiore 17-21 settembre 1989) (erscheint 1991).

${ }^{4}$ A.a.O. $512 \mathrm{ff}$.

5 Fol. $1^{\text {r: }}$,Hunc librum concordanciarum Joachim. noui ac ueteris testamenti. Dedit Venerabilis / pater et Dominus dominus Ildebrandinus episcopus paduanus (sic!) conuentui Beati Antonii de padua. / ipso uiuente. Sub modo et forma qui in fine libri continetur. " Fol. $139^{\text {ra }}$ notiert der Schreiber, daß er zu geringen Lohn erhalten habe: „Huius erat precium scripture tam male strictum / Quod potui miserum uix hinc acquirere uictum. / Supplico si placeat. aliqualis gracia fiat.“ Der Scriptor war also kein Mönch. - Fol. 139 ${ }^{\text {rb }}$ steht der Schenkungsvertrag: „Anno domini millesimo. CĆC. Lij. die xxxvja ${ }^{\mathbf{a}}$ / septembris. Venerabilis pater et dominus / dominus Ildebrandinus dei gratia episcopus pa/duanus nomine donationis inter uiuos. De/dit et donauit hunc librum. Fratri Johanni de / monte sancto tunc sacri conuentus Beati Antonii / guardiano. recipienti nomine et uice dicti con/uentus. hiis condicionibus appositis. Videlicet quod (in) / usum dicti libri sibi retinuit in uita sua tantum. / Ita quod quandocunque predictus dominus uellet dictum librum. / Guardianus et fratres dicti conuentus ipsum sibi concedere / teneantur. Et quod predictum librum non possint ipsi / fratres. uendere. impignorare alicui obligare. / uel appropriare. sed semper remaneat in supradicto sacro loco ad comodum et utilitatem fratrum / inhibi comorantium et quod si secus factum fuerit / exnunc prout extunc ipsos fratres dicto libro priuat / et priuatos esse uult et mandat. ac ipsum donat / et dat capitulo canonicorum ecclesie maioris de / padua. Actum in palatio sepedicti domini in camera sua. Presentibus Domino Nicolao / abbate Sancte Justine de padua. [Lücke] / [Lücke] priore cruciferorum. et fratre Antonio de cortarodulo. Et aliorum. etc." L. Luisetto, G. Abate, Codici e manoscritti della Biblioteca Antoniana di Padova (Vicenza 1975) 290 ff.; vgl. Art. „Conti, Ildebrandino“, in: Dizionario biografico degli Italiani 28 (1983) 438-440 (B. G. Kobl), L. Luisetto, Ildebrandino Conti e i suoi codici donati alla Biblioteca Antoniana di Padova, in: Almanacco dei Bibliotecari Italiani (1952) 195-198; Antonio Maria Adorisio, Codici latini calabresi (Roma 1986) 27.

${ }^{6}$ Fol. 139va.: „Egregius legum doctor dominus Paganinus / a sala. Asignauit hunc librum fratri Bartho/lomeo de sancto Georgio. quem habuit ex sua solici/tudine. de libris inuentis post mortem domini Ja/cobi de sancta cruce. Quem librum dedit venera/bilis pater et dominus yldebran- 
Die Geschichte der Lektüre der Codices aufzuhellen, für die Besitzvermerke und Randnotizen im besten Fall karge Hinweise geben, ist also eine weitere Aufgabe, die zu unserem Thema gehört. Sie ist nicht in Angriff genommen, und ich kann nur sporadische Notizen mitteilen. Die Handschriften sind noch nicht einmal für die Edition der in ihnen enthaltenen Joachim-Texte auch nur annähernd genügend ausgewertet worden. Es gibt, so kann man mit Gewißheit sagen, kaum eine einzige Edition eines Werkes Joachims, die kritischen Ansprüchen voll genügte. Bestenfalls kann man sagen, daß „lesbare Texte“ vorliegen. Ediert ist fast alles, aber wie! Die ganze reichhaltige Joachiminterpretation ruht damit auf einem in vielen Einzelheiten unsicheren Grund und kann der Forschung nur deshalb genügen, weil sie literarhistorisch-philologisch allzu bescheiden ist und es so genau eigentlich nicht wissen will ${ }^{7}$.

Ich lasse es, weil mehr hier nicht möglich ist, bei einem „Entertainment“ mit einigen Details bewenden, die sich mir bei der Arbeit an den Handschriften beiläufig ergeben haben.

Zunächst die Zahl der Handschriften. Reeves führt für die echten Werke etwa 50 Handschriften auf - ein im ganzen schmaler Bestand für drei Jahrhunderte, auch wenn er sich vermehren läßt und die Textgeschichte auf einen beträchtlichen Eisberg unter Wasser schließen läßt. Von diesen ca. 50 Handschriften wird etwa die Hälfte auf das 14. und 15. Jahrhundert datiert, was nur approximativ richtig sein wird; eher gehören einige weitere Codices, die dem 13. Jahrhundert zugewiesen sind, auch ins 14. Jahrhundert.

Ich lasse jetzt die sechs Handschriften beiseite, die mit Sicherheit in die erste Hälfte des 13. Jahrhunderts gehören, ja bis an den Anfang des Jahrhunderts gerückt werden müssen ${ }^{8}$. Damit ergibt sich jedenfalls ein Übergewicht der erhaltenen Überlieferung von der 2. Hälfte und eher vom Ende des 13. Jahrhunderts an. Dafür mögen zwei Codices als Beleg dienen: der eindeutig aus dem Kreis der italienischen Franziskanerspiritualen stammende Miscellaneakodex Vat.lat. 3822 - der auch viel Pseudo- und

\section{Fortsetzung Fußnote von Seite 50}

dinus episcopus paduanus / conuentui paduano. sicut continetur in principio. et in fine / dicti libri." Zu dem 1352-1388 tätigen Rechtsgelehrten Paganinus a Sala, der an der Universitāt lehrte und in diplomatischer Mission für die Carrara wirkte, aber schließlich als Verräter an den Mailänder Visconti gehängt wurde, vgl. Andrea Gloria, Monumenti della Università di Padova II (Padova 1888) Nr. 318-325. Ein Abt Bartholomāus von S. Giorgio (Venedig) wird im Umkreis Ildebrandinos als Visitator der Benediktinerklöster 1339 genannt. Paolo Sambin, Un amico del Petrarca, Ildebrandino Conti e la sua attività spirituale e culturale, in: Miscell. di Studi e memorie della Dep. di storia patria per le Venezie 8 (1952) 3-56, auf 15.

7 Herbert Grundmann, Studien über Joachim von Floris (Leipzig 1927, Stuttgart ${ }^{2} 1985$ ) hat zwar die allgemeine Zuverlässigkeit der venezianischen Drucke festgestellt; das bezieht sich aber nur auf die Authentizität und den allgemeinen Inhalt, nicht auf die Einzeltexte, bei denen zahllose Korruptelen vorliegen. „Lesbar" sind auch die weiteren neuedierten Texte - mit Ausnahmen! -, aber eben nicht durchweg im Detail zuverlässig. Vgl. demnächst mein Verzeichnis der Werke und Editionen im Artikel „Joachim abbas de Flore“ des Repertorium Fontium Historiae Medii Aevi VI und in: Florensia 3 (1989, erscheint 1991).

${ }^{8}$ Vgl. $K .-V$. Selge, L'origine delle opere di Gioacchino da Fiore, in: L'attesa della fine dei tempi nel Medioevo (Quaderni dell'Istituto Storico Italo-Germanico in Trento 28, 1990) 129 Anm. 91, und unten Anm. 29. 
Nachjoachimitisches enthält ${ }^{9}$ - und der komplett wohl aus einem französischen Original abgeschriebene Dresdner Codex, der zwei Schriften Joachims als „Vorrede“ und als „2. Buch des Evangelium aeternum“ oder „Evangelium des HI. Geistes“ bezeichnet $^{10}$. Das lokalisiert dieses neue Interesse an Joachims Endzeitexegese einigermaßen genau. Es ist aber nicht der einzige Überlieferungsträger; denn die Mehrheit der Handschriften aus dieser und der Folgezeit trägt ein solches Signum nicht und entstammt also eher einem normalen, sicher vorwiegend monastischen Ủberlieferungsstrom, an dem jedenfalls der Zisterzienser- und der Franziskanerorden ${ }^{11}$ in seiner konventualen Mehrheit und später auch der Karmeliterorden beteiligt sind. Wie weit der Augustinereremitenorden, aus dem die Drucke hervorgehen, beteiligt ist, läßt sich bisher nicht erkennen. Und da die Überlieferung aus dem Anfang des 13. Jahrhunderts meist nicht die Archetypen der späteren Texte bietet ${ }^{12}$, darf man sich von den Handschriftenzahlen auch nicht zu falschen Schlüssen verleiten lassen: Es hat in der ersten Jahrhunderthälfte eine große Menge Joachim-Handschriften gegeben, die die Texte in dem normalen Überlieferungsstrom im Florenser-, Franziskaner- und Zisterzienserorden der Folgezeit überliefert haben; vielleicht hat es einen Produktionsknick und auch Vernichtung von Texten in der Zeit der Kämpfe um das Evangelium aeternum und um die „Spiritualen“ gegeben, die dann ihre eigene Überlieferungstradition schufen. Aber ob irgendeine erhaltene spätere Handschrift aus den genannten Handschriften, die diesem Spiritualenmilieu entstammen, abgeschrieben worden ist, weiß ich nicht. Ildebrandinus von Padua jedenfalls hatte um 1350 eine ausgezeichnete Vorlage für seine "Concordia“, die auf alte Tradition zurückgehen mag und keine Fortspinnung des Textes im Geist der Spiritualen enthält, wie sie sich in der genannten vatikanischen Handschrift findet.

Daß die Joachitica im 14. Jahrhundert fromme und gelehrte italienịsche Bürger und einzelne konventuale Franziskaner interessierten und ein wechselseitiger Austausch

9 Jeanne Bignami-Odier, Notes sur deux manuscrits de la Bibliothèque Vaticane, in: Mélanges d'Archéologie et d'Histoire 54 (1937) 211-241. Aus Spiritualenkreisen stammt, wie ich nach einer erneuten Durchsicht aufgrund verschiedener Indizien glaube, auch der ebenfalls in sehr kleiner Schrift geschriebene Pariser Codex B.N. lat. 10453, der die „Concordia“ enthält. Vgl. den Christuskopf auf f. $65^{\text {va }}$ (zu einem Text, der auf den künftigen Ordo verweist) und die in größerer Schrift abgefaßten Passagen auf f. $89^{\text {rb/va }}$, in denen von den endzeitlichen Spiritualen und der Verheißung eines neuen Herzens und eines neuen Geistes die Rede ist.

10 Sächsische Landesbibliothek A 121. - Herbert Grundmann, Neue Forschungen über Joachim von Fiore (Marburg 1950) $27 \mathrm{f}$. und oben Anm. 8.

"Aus der Bibliothek von Clairvaux stammt der große Codex in Troyes, Bibliothèque municipale 249, der u.a. zwei ursprünglich selbständige Codices der „Concordia“ und der „Expositio in Apocalypsim" (aus dem 13. Jahrhundert) vereinigt. Geschrieben sind die Texte vermutlich an anderem Ort. Das besondere - vorwiegend kritische - Interesse des Zisterzienserordens an der Produktion Joachims, das auch hinter der Verurteilung der Trinitätslehre Joachims durch das 4. Laterankonzil 1215 stehen dürfte, ergibt sich aus der gesamten Frühgeschichte Joachims; vgl. nur den heftigen Angriff des ehemaligen Sekretärs und Biographen Bernhards von Clairvaux, Gaufrid von Auxerre, gegen Exegese und Person Joachims. Herbert Grundmann, Ausgewählte Aufsätze, Teil 2: Joachim von Fiore (Stuttgart 1977) 324-338.

12 Eine Ausnahme scheint der durchkorrigierte Text des „Psalterium decem cordarum“ im Cod. 322 der Biblioteca Antoniana in Padua zu sein, der aus dem 1. Viertel des 13. Jahrhunderts stammt und ausdrücklich als „Exemplum“, d.h. als Musterexemplar, bezeichnet wird (fol. $42^{\text {rb }}$ ). 
stattfand - wie es in dem geschilderten Fall aus Padua gewesen zu sein scheint -, zeigt auch schön ein Vermerk in einer Handschrift des Concordienbuches in der Biblioteca Laurenziana, die aus dem Franziskanerkonvent von S. Croce stammt (plut. 8 dex. 10): „Am 16. Juli 1393 hat Herr Domenico Allegri, gesunden Geistes doch körperlich hinfällig, mir, dem Bruder Thedaldus, in Gegenwart des Notars der Arte della Lana dies Buch Joachims der Concordia des Alten und Neuen Testaments geschenkt, damit ich für seine Seele zu Gott bete; und ich habe mich dazu frei angeboten und verpflichtet, weil ich schon lange den Wunsch gehegt hatte, dies Buch zu haben, das ich mit großer Mühe endlich zu lesen gelernt habe."13

Die Pointe liegt auch darin, daß dieser Thedaldus della Casa, ein wohlbekannter Abschreiber und Büchersammler aus dem Umkreis Coluccio Salutatis, offenbar den Codex früher selbst im Auftrag des Herrn Allegri geschrieben hat. Er hat sich offenbar lebhaft für Joachim interessiert; denn ein anderer Codex von S. Croce (plut. 9 dex. 11) enthält in seiner Schrift die Inhaltsangabe: „Joachim, Super Jeremiam“ usw., und eine Notiz aus dem Jahr 1406 sagt: „Dies Buch war ad usum“ - d. h. praktisch: es gehörte, es war ihm geschenkt worden - „des frater Thedaldus de Casa; er hat es zu Lebzeiten der Bibliothek des Florentiner Konvents der Fratres minores übergeben "14 .

Ich gebe ein neues Beispiel. Im Generalarchiv der Karmeliter befindet sich ein Miscellaneakodex, der z.T. um 1480 in Rom ,auf dem Campus florum im Hause des Herrn Magister Petrusleonis von Spoleto“, der „aller Artes liberales peritissimus“ genannt wird, geschrieben wurde. Es handelt sich um den bekannten Freund Marsilio Ficinos und Leibarzt Lorenzos des Prächtigen; er hat in dem Codex auch seine Marginalien hinterlassen. Ich komme hierauf noch einmal zurück. Der Codex ist aber aus einer Vorlage vom Anfang des 14. Jahrhunderts abgeschrieben; denn er enthält neben sechs Werken Joachims von Fiore vor allem eine Reihe von Hauptwerken Arnolds von Villanova, ein Fragment von Ubertino von Casales „Arbor vitae crucifixae Jesu“, weitere Joachitica und Exegetica und große Teile von Hauptwerken des Pariser Bischofs und Magisters Wilhelm von Auvergne aus dem 13. Jahrhundert. Die Handschrift zeigt eine einheitliche Schrift, und keiner der Texte ist später als Anfang des 14. Jahrhunderts zu datieren, das in einem der Antichristtexte Arnolds als das „Saeculum quod nunc currit" bezeichnet wird: in ihm soll nach einem Ende des 13. Jahrhunderts erschienenen Propheten der „maximus Antichristus“ erscheinen. Die Abschrift

13 Fol. $168^{\mathrm{v}}$ : „Nota quod Anno domini MCCCLXXXIII. die XVI. Julii ser Dominicus Allegri, sanus mente sed corpore languens presentibus ser francisco [Lücke] notario artis lane et nicholo delbene michi fratri thedaldo donauit et libere largitus est hunc librum concordie Joachim ueteris et noui testamenti, ut ego pro eius anima rogarem deum. et ego ad hoc me libere obtuli et obligaui. quia a diu desideraueram hunc librum habere. quem quamuis difficulter. tandem tamen legere didici." Über Thedaldus als Abschreiber antiker, humanistischer und theologischer Texte vgl. schon G. Voigt, Die Wiederbelebung des classischen Alterthums I $\left({ }^{3} 1893={ }^{4} 1960\right) 397 \mathrm{f}$.

${ }_{14}$ Fol. II ${ }^{\mathrm{vb}}$ : „In hoc libro continetur abbas Ioachim super Ieremiam prophetam. De ultimis tribulationibus. De articulis fidei ad fratrem Iohannem. N. LXXXXII." Das ist offenbar die Lokalisierung im Armarium des Florentiner Konvents. - Daß Thedaldus della Casa auch in der Überlieferung der Werke Ockhams erscheint, vermutet zumindest $A$. M. Bandini, Catalogus codicum latinorum Bibliothecae Mediceae Laurentianae IV (Florentiae 1777) col. $716 \mathrm{f}$. (zu Ms. Florenz, Bibl. Med. Laur., S. Croce, plut. XXXVI dextr., c. 11); freilich bleibt das problematisch. 
von Joachims "Liber exhortatorius Iudeorum“ ist in dieser Vorlage am 2. März 1304, dem 1. Tag der Fastenzeit, beendet worden, und die Abschrift 180 Jahre später gibt das getreulich wieder ${ }^{15}$. Diese erste Vorlage dürfte, wenn in ihr das Jahr nach dem Calculus Pisanus gezählt wurde $(1304=25.3 .1304-24.3 .1305)$, in Mittelitalien entstanden sein. Hat Pierleoni sie in Pisa gefunden, wo er jahrelang Lehrer der Medizin war? ${ }^{16}$

Ein weiteres Beispiel. Es gibt neben der endgültigen Einleitung, dem „Liber introductorius“ zu Joachims Apokalypsenkommentar, vier vollständige Zeugen für eine erste ausführliche Fassung dieser Einleitung, „Encbiridion super Apocalypsim“ genannt ${ }^{17}$. Sie liegen in Paris (2. Hälfte 13.Jh.), Pavia (Anfang 13.Jh.), Weimar (13.Jh.) und Rom, hier in einer Handschrift aus dem Besitz der Königin Christina von Schweden. Diese letztgenannte Handschrift stammt aus Böhmen und ist datiert. Der dem Enchiridion vorangehende Text, der Werke Augustins und das „Elucidarium“ des Honorius Augustodunensis enthält, an dessen Ende die Schriftgelehrsamkeit und Verführungskraft des Antichrist besonders für die Mönche beschrieben wird, endet mit der Angabe: „Im Jahr 1346 sind diese Hefte geschrieben worden per manus conradi, indignissimi eruditoris parvulorum scole pilgrimensis civitatis“, also des Lehrers der Stadtschule von Pelhrimov ${ }^{18}$. Die Schrift ist extrem schnörkelreich, steil und eckig, großformatig und reich wie der ganze Prachtkodex, und dieselben Charaktere weist auch das anschließend von ähnlicher Hand geschriebene „Enchiridion“ Joachims auf ${ }^{19}$. Es bestand ein Bedarf an Kurzfassungen des riesigen Apokalypsenkommentars, von dem uns ja auch viele Exzerpte und eine ganze eigene Kurzfassung überliefert ist ${ }^{20}$. Daher also die Sonderüberlieferung des eigentlich nach Abschluß des Hauptkommentars überflüssigen „Enchiridion“. Man kann vielleicht auch einen Zusammenhang sehen zwischen der Paduaner "Concordia“ des Bischofs Ildebrandinus Conti um 1340/50 und dem böhmischen Prachtkodex mit dem Enchiridion. Es ist die Zeit Karls IV. und des frühen Humanismus, der das „Avignoneser Exil“ als babylonisches

${ }^{15}$ Es dürfte sich nach dem hier wohl verwendeten Calculus Pisanus (Jahresanfang 25. März des Vorjahres) um den 2. März 1305 handeln, der in diesem Jahr der Vorabend des Aschermittwoch war.

${ }^{16}$ Vgl. meine Beschreibung des Codex: Un codice quattrocentesco dell'Archivio Generale dei Carmelitani, contenente opere di Arnaldo da Villanova, Gioacchino da Fiore e Guglielmo da Parigi, in: Carmelus 36 (1989) 166-176 und 37 (1990) 170-172. - Pierleoni von Spoleto hat sich 1475, 1478-1482 und 1487-1490 vorwiegend in Rom aufgehalten. Dazwischen lehrte er in Pisa, anschließend in Padua. L. Guerra-Coppioli, M. ${ }^{\circ}$ Pierleone da Spoleto, medico e filosofo. Note biografiche con documenti inediti, in: Bollettino della Regia Deputazione di Storia Patria per l'Umbria 21 (1915) 387-431. - Das im Codex angegebene Datum dieser Abschrift ist Dienstag, 4. Juli 1480.

${ }_{17}$ Unzureichend ediert von Edward Kilian Burger, Joachim of Fiore. Enchiridion super Apocalypsim (Studies and Texts 78, Toronto 1986).

${ }_{18}$ Biblioteca Vaticana, Reg. lat. 132, fol. $48^{\text {rb }}$. Andreas Wilmart, Codices Reginenses latini I (Città del Vaticano 1937) $308 \mathrm{ff}$.

19 Die ungewöhnliche Schrift gleicht der dreier in Prag geschriebener Briefe des Cola di Rienzo von 1350; Faksimile in: Konrad Burdach (Hg.), Vom Mittelalter zur Reformation II, 3 (Berlin 1912) Tafel III.

${ }^{20}$ Die sogenannte „Apocalypsis nova“; vgl. mein oben Anm. 7 genanntes Werkverzeichnis und oben Anm. 10. 
Exil der Kirche bezeichnete und damit ein von den Franziskanerspiritualen gebildetes Stichwort mit kirchenkritischer Spitze aufnahm ${ }^{21}$.

Im vergangenen Jahr bin ich nach Basel und Köln gereist, um mir die dortigen Handschriften von Joachims Apokalypsenkommentar anzusehen. Der Basler Codex ist 1434 vom dortigen Kartäuserkonvent erworben und vermutlich kurz zuvor am selben Ort geschrieben worden ${ }^{22}$. Der Kölner Codex entstammt dem dortigen Karmeliterkonvent und ist, wie die Textfehler zeigen, eine Abschrift des Basler Codex. Diese Abschrift ist am 9.2.1435 - sicher in Basel - von dem Kölner Studenten Heinrich von Osnabrück, der noch jahrzehntelang später als Schreiber bezeugt ist, abgeschlossen worden ${ }^{23}$. Der Besitzer des Codex, Heinrich de Iudeis, ein Kölner Rechtsgelehrter und Pfarrer, der ihn später den Karmelitern vermacht hat, war 1434 auf dem Basler Konzil. Er hat sich den Text wohl von Heinrich von Osnabrück anfertigen lassen und ihn später dem Konvent des Abschreibers vermacht ${ }^{24}$. Die Textemendationen des Kölner Codex zeigen nun, daß in Basel weitere Exemplare des Apokalypsenkommentars zur Verfügung standen, und eine Notiz zum Anfang des Prologs beweist, daß auch Joachims „Enchiridion“, die Erstfassung der Einleitung, 1434 in Basel vorhanden war $^{25}$. Eine Marginalie zum Thema des Büchermarktes des Basler Konzils und ein Indiz dafür, daß der aus dem Besitz Pierleonis von Spoleto stammende römische Codex, der mit Sicherheit im 18. Jahrhundert bei den römischen Karmelitern von S. Maria Traspontina vorhanden war $^{26}$, ihnen schon jahrhundertelang gehört haben kann und eventuell ein im ganzen 15. und 16. Jahrhundert bestehendes Interesse in diesem Orden an apokalyptisch-exegetischen Texten, die abseits der kirchlichen Normaleschato-

${ }^{21}$ Vgl. den berühmten Brief (Ep. sine nomine 8) Petrarcas an Ildebrandino Conti aus Avignon 1351 und die Zeugnisse über das Interesse an Joachitica im Kreis Cola di Rienzos, Briefwechsel des Cola di Rienzo, hg. v. Konrad Burdach u. Paul Piur, 4 und 5 (Vom Mittelalter zur Reformation, hg. v. Burdach, II,4, 1912 und II,5, 1929) passim.

22 Basel, Universitätsbibliothek B III 19, fol. $240(236)^{\mathrm{vb}}$ : „1434 Liber Cartusiensium Bas.“; Deckblatt $A^{v}$ Besitzvermerk: „Iste liber est Carthus. bas. comparatus sub priore et patre albertho buer de traiecto inferiori / et continet Joachim super apocalipsim“ (gleichzeitige Handschrift).

${ }^{23}$ Köln, Stadtarchiv W 267, fol. 168 : „Explicit expositio libri Apocalipsis Joachim abbatis finita anno d. $\mathrm{M}^{\circ} \mathrm{CCCCXXXV}$ ipso die s. Apolonie virg. et mart. per H. de Osnabrugis."

${ }^{24}$ Besitzvermerk des 15. Jahrhunderts auf der Innenseite des Vorsatzblattes. Joachim Vennebusch, Die theologischen Handschriften des Stadtarchivs Köln, Teil II: Handschriften der Sammlung Wallraf (Köln-Wien 1986), mit Abbildung 8. Über den Abschreiber Heinrich von Dissen (bei Osnabrück) vgl. NDB 3 (1957) $743 \mathrm{f}$. Er ist 1415 in Osnabrück geboren; 1437 legt er in der Kölner Kartause St. Barbara die Profeß ab, 1484 ist er in Köln gestorben. Er ist als Abschreiber bis nach 1480 bezeugt. - Der getaufte Jude Heinrich de Judaeis ist 1421 (und erneut 1434?) in Köln immatrikuliert, Doctor decretorum, Pfarrer von St. Martin 1431-1473, 1434 in Basel. Er hat den Kölner Kartāusern auch die Decreta concilii Basiliensis geschenkt: Hermann Keussen, Die Matrikel der Universität Köln I (Bonn 1928) Nr. 129,11 und 182,27.

${ }^{25}$ Fol. $1^{\text {va }}$, „Alii habent sic. Prologus in enchiridion Joachim super apocalipsim. Quam propensioribus studiis a viris catholicis et orthodoxis certatum est, ut lactentis et adhuc rudis ecclesie fundamenta iacerent et eius deo dicatam infanciam lactis potu nutrirent illorum que per cursum ipsum ad nos derivata sunt opera celestium etc." Vgl. Burger (oben Anm. 17) S. 9 Z. 1-5 und den abweichenden endgültigen Text der Expositio in Apocalypsim (Venedig 1527, Neudruck Frankfurt a. M. 1964) fol. $1^{\mathrm{vb}}$.

${ }^{26}$ N. Antonio, Bibliotheca Hispana Vetus (Madrid 1788) 118. Die Kenntnis dieses Zeugnisses verdanke ich Robert Lerner. 
logie lagen, bezeugt. Hiervon aber wäre es nicht schwer, eine Brücke zu schlagen zu dem Interesse an Bibelexegese, Judaica und Apokalypse, das sich in den Marginalien Pierleonis von Spoleto - dem Freunde Marsilio Ficinos und florentinischen Zeitgenossen Savonarolas - sowohl zu dem römischen Karmeliterkodex wie zum aus dem 13. Jahrhundert stammenden Manuskript des Apokalypsenkommentars in der Vatikanbibliothek zeigt. Ich wollte auf diese Marginalien in den Büchern Pierleonis noch einmal zurückkommen ${ }^{27}$. Ihn interessiert die Methode der Schriftauslegung. Viele seiner Notizen betreffen die Hermeneutik ${ }^{28}$ : „Optima regula interpretandi sacras litteras“ (Chig. 39\%). Er notiert Historisches: „Zur Zeit Joachims haben die Sarazenen viele Spanier getötet“ (87》). Ihn interessiert Hebräisches - „Adonaj - das Tetragrammaton“ $\left(26^{r}\right)$ : „Adonaj ist mein Name, heißt: mein Name ist Dominus“. Er notiert sich, daß eine Passage „gegen die Astronomen" gerichtet sei, und ihn interessiert Kirchenkritisch-Eschatologisches.

Es handelt sich bei diesem Apokalypsenkommentar aus der Vaticana um eine der ältesten Joachim-Handschriften überhaupt, wahrscheinlich aus dem Besitz des Klosters S. Giovanni in Fiore ${ }^{29}$. Nur ein Leser hat 200 Jahre vor Pierleoni seine Notizen in ihr hinterlassen, und sie sind von ganz anderer Art. Da hat ein Erzbischof, wohl von S. Severina, die Texte auf offenbar heftige Konflikte bezogen, in denen er mit Suffraganen und Fürsten stand, und Recht und Unrecht zu finden gesucht: „gegen die Dialektik, gegen die Rechtsgelehrten, gegen den Bischof von S. Leone, für mich, den Erzbischof", aber auch: "gegen mich, den Erzbischof“, gegen den Grafen von S. Agata, den Herzog von Andria, und „contra karolum“ - gegen den König Karl. Er, der Erzbischof, muß, bedrängt, bußfertig bleiben, damit er nicht von Gott gänzlich vernichtet werde $^{30}$. Das ist eine ziemlich authentische Weise, Joachim zu lesen, im Süditalien des

${ }^{27}$ José Ruysschaert, Nouvelles recherches au sujet de la bibliothèque de Pier Leoni, médecin de Laurent le Magnifique, in: Académie royale de Belgique, Bulletin de la Classe des Lettres et des Sciences morales et politiques, $5^{\text {e }}$ série, t. 46 (1960) 37-65 (Tafeln II b, III a/b); ders., Constantino Gaetano, O.S.B., chasseur de manuscrits, in: Mélanges Eugène Tisserant VII (Studi e Testi 237, Città del Vaticano 1964) 261-326, auf $272 \mathrm{f}$. und 289 (Nr. 231: Codex Chigi A.VIII. 231, hier jedoch irrig auf das 14. Jahrhundert datiert); Giorgio Radetti, Un' aggiunta alla biblioteca di Pierleoni da Spoleto, in: Rinascimento 16 (1965) 87-99.

${ }^{28}$ Ich gebe hier nur Belege aus den Randnotizen Pierleonis zum Apokalypsenkommentar Joachims, Bibl. Vat., Chig. A. VIII. 231.

${ }_{29}$ Der Schreiber ist mit dem des Codex der Bibl. Corsiniana (Rom), 41 F 2, identisch, der einen Besitzvermerk des Klosters S. Giovanni in Fiore enthält. Die Identität der Handschrift ist von Fabio Troncarelli festgestellt worden und wurde mir auch auf meine Bitte von Emmanuele Casamassima bestätigt. $F$. Troncarelli, Nuove reliquie dello "Scriptorium“ di Fiore, in: $A$. Crocco (Hg.), L'Età dello Spirito e la fine dei tempi in Gioacchino da Fiore e nel Gioachimismo medievale (Atti del II congresso Internazionale di Studi Gioachimiti 6-9 settembre 1984, S. Giovanni in Fiore 1986) 319-329, auf $319 \mathrm{f}$. Die weitergehende kulturhistorische Interpretation dieser Schrift, der ein quasi geschichtstheologisch-trinitarischer „kaleidoskopartiger“ Charakter zugeschrieben wird, scheint mir übertrieben zu sein. Vgl. die Abbildungen in $A . M$. Adorisio, Codici latini calabresi (Rom 1986).

${ }^{30}$ Diese Randbemerkungen bedürfen eines eigenen Studiums; sie beginnen auf fol. $1^{\mathrm{b}}$ (,De impiis", „De veritate contra infideles") und enthalten Personenangaben, z. B. $5^{\text {tb. }}$,pro archi(episcopo)", „marchio et co(mes) mo(ntis) al(ti); $7^{\mathrm{va}}$ : „, Rex Ka(rolus)“; $50^{\mathrm{va}}$ : „,ontra Io. de plan. per totum"; $79^{\text {bb }}$, „contra ep(iscopum) Sancti Le(onis), pro me archiepiscopo“; $60^{\text {vb }}:$,pro domo s(ancte) 
späten 13. Jahrhunderts - eine andere Art als die mehr gelehrt-kirchenkritische Lektüre durch Bürger, Mönche, Humanisten im 14. und offenbar verstärkt im Umkreis von Rom, Florenz, Venedig am Ende des 15. Jahrhunderts. Von den für diese späte Zeit angeführten Zeugnissen führt eine Brücke zum Interesse des Augustinereremitenkreises um den Ordensgeneral Aegidius von Viterbo am Druck dieser Werke ab 1516, ferner zur Anfertigung der beiden prächtigen Handschriften der beiden Hauptwerke Joachims, des „Liber Concordiae“ und der „Expositio super Apocalypsim“, die in den achtziger Jahren des 15. Jahrhunderts in Rom auf venezianischem Papier und vielleicht von einem deutschen Scriptor geschrieben wurden und heute in der Biblioteca Casanatense liegen ${ }^{31}$, und schließlich auch zu einem letzten Beispiel für das Interesse des frühen 16. Jahrhunderts an den apokalyptischen Zeichen der Zeit, mit dem ich diese fragmentarische Skizze abschließen will.

In der Stadtbibliothek von Todi liegt ein schöner alter Codex des Apokalypsenkommentars Joachims vom Ende des 13. oder Anfang des 14. Jahrhunderts ${ }^{32}$. Die Akten des Rates der Stadt Todi enthalten einen Schriftwechsel ${ }^{33}$. 1522 erbat sich der Florentiner päpstliche Kardinalpönitentiar, Kardinal Lorenzo de' Pucci, von den

\section{Fortsetzung Fußnote von Seite 56}

s(everine?) contra ali(os)“; $61^{\text {ra }}$ : „contra comitem S. Agathes“; $15^{\text {va }}$ : „contra ka(rolum) “; 66 ${ }^{\text {va }}$ : "contra dyalecticam“, „contra legis peritos“. Persönlich spricht der Erzbischof sich an zwei Stellen aus: $46^{\mathrm{rb}}$ "Non est magnum habere caritatem in prosperis nisi quis fortis maneat in adversis“ und $60^{\mathrm{rb}}$ "contra me archiepiscopum" (Glosse zu dem Text: „ne forte ex aliqua sui parte destitutus corruat et putans se esse aliquid redigatur ad nichilum"). Offenbar steht ein Erzbischof von S. Severina im Konflikt mit seinem Suffragan, dem Bischof von S. Leone, und verschiedenen Potentaten. Möglicherweise handelt es sich um Rogerius Stephanicius (1272-1295, 1288-1295 vertrieben), vgl. Norbert Kamp, Kirche und Monarchie im staufischen Königreich Sizilien I,2: Apulien und Kalabrien (München 1975) Nr. 119 S. 891. Er wurde 1296 noch Erzbischof von Cosenza und fiel im selben Jahr im Kampf gegen die Aragonesen.

${ }^{31}$ Casanat. 1411 (Expositio super Apocalypsim) und 1412 (Concordia). Am unteren Rand des Cod. 1411 steht in alter Handschrift: „Ad usum S. Mariae Pacis de Urbe“. Das Wasserzeichen findet sich auf Papier des Staatsarchivs Venedig wieder und weist auf die Jahre 1475 bis 1495. Vgl. C. M. Briquet, Les Filigranes I (Leipzig $\left.{ }^{2} 1923\right) 40$ und Nr. 461. Antonio Maria Adorisio (Rom) meint, es handle sich um zwei in Rom tätige deutsche Scriptoren, und verweist auf Briquet Nr. 45963.

32 Todi, Bibl. com. ms. 43. Lorenzo Leoni, Inventario dei Codici della Comunale di Todi (Todi 1878).

${ }^{33}$ Stadtarchiv Todi, Liber Reformationum Iohannis Fabritii Ofredutii de attis de Tudeto anni $M^{\circ}$ CCCCCXXII (sic!) p. $67^{\mathrm{v}}$ : "Copia litterarum R.mi Card. lis S. torum quatuor." Der Originalbrief ist erhalten unter der Signatur: Lettere ai Priori, fasc. 1518-22, Nr. 227: „Magnifici Domini, Amici nostri charissimi. Salutem. Alias quondam Petrus de' Todi concivis vester librum habuit Abbatis Joachim, super apocalypsi ex libraria Domus S.ti Francisci Tudertine, ad certum tempus, eumque accommodavit Magistro Petro Galatino ordinis minorum professori, Capellano nostro. Cum autem dictum tempus iam affluxerit, nosque librum ipsum ad manus nostras receperimus, ut eum transcribi faciamus, conveniens fore duximus, id vobis qui dicte domus patroni estis per litteras nostras significare in fide nostra promittentes, quod quamprimum liber ipse transcriptus fuerit, eum ad vos transmittemus curabimusque ut quantocius fieri poterit, transcribatur. Et bene valete: Rome ex Poenitentaria apostolica. Die XXV', mensis Junii MDXXII ${ }^{\circ}$. Vester tanquam frater Laur. Car.lis Sanctorum Quattuor manu propria.“ Die Adresse des gefalteten und gesiegelten Briefes lautet: „Magnificis Dominis Prioribus populi et comunis Civitatis Tudertine, Amicis nostris charissimis." 
Priori del popolo von Todi eine Verlängerung der Ausleihe dieses Codex, der den Franziskanern von S. Fortunato gehörte und früher von dem römischen Senator Petrus de Corradis, einem Bürger von Todi, nach Rom entliehen worden war. Dieser hatte ihn seinerseits dem Franziskaner Petrus Galatinus, dem orientalistisch gebildeten Humanisten und Freund Johannes Reuchlins, überlassen, und nach mehreren Bemühungen um Rückgabe war der Codex dem Kardinal übergeben worden, der ihn abschreiben lassen wollte ${ }^{34}$.

Petrus Galatinus aber verfaßte in den Folgejahren eine Reihe von - bis heute ungedruckt und fast unstudiert im Vatikan liegenden - Büchern, darunter einen riesenhaften eigenen Kommentar zur Apokalypse, den er keinem Geringeren als Kaiser Karl V. widmete ${ }^{35}$. Der Kaiser wird das Opus nie zu Gesicht bekommen haben. Am Druck apokalyptischer Werke bestand in Rom 1540, als Petrus Galatinus starb, begreiflicherweise keinerlei Interesse mehr. Die drei Jahrhunderte lang schlafenden, aber auch immer einmal sich räkelnden, aufwachenden und bellenden Hunde der ekklesiologischen Unruhe, die die Apokalyptik seit der Zeit Joachims in die Christenheit getragen hatte, waren vernehmlich aufgewacht und hatten sich zur Meute zusammengerottet. Luther hatte den endzeitlichen „Antichrist“ im römischen Papsttum identifiziert und geißelte ihn in der bekannten Weise bis zu seinem Tode. Genügend Menschen auch in Italien bis in hohe Kreise auch der Kirche - z. B. der Bischof Pierpaolo Vergerio - ließen sich davon beeindrucken, und so wurde die Eschatologie besser gegenreformatorisch domestiziert. Ketzerrecht und gesunde Lehre für eine diesseitige, dauernde Kircheninstitution, die sich pastoral reformierte, waren die römischen Waffen für die neue Zeit. Vom Antichrist redeten die Protestanten, besonders auch die westeuropäischen Calvinisten, die anders als die deutschen Lutheraner nach 1555 jahrzehntelang im Kampf standen. Aber auch in der protestantischen - besonders lutherischen - Orthodoxie nach 1560 verlagerte sich das Gewicht auf die reine Lehre für das Diesseits und auf die Hoffnung für die jenseitige Ewigkeit nach dem Tode und am fernen Ende der Tage. Die apokalyptischen, teils gelehrten, teils kirchenkritischen und kirchenreformerischen Interessen des Spätmittelalters bekommen in der Perspektive des 16. Jahrhunderts fast etwa Idyllisches. Aber es ist klar, daß ihre Existenz neben der offiziellen scholastisch-kirchlichen Lehre von den letzten Dingen ein Fer-

${ }^{34}$ Über die Rückgabeversprechungen finden sich im Stadtarchiv Todi weitere Eintragungen, auf die mich Roberto Rusconi hinweist: 10./11. November 1521 (Memorie della chiesa e convento di San Fortunato, Arm. IV, cas. XII; Riformanze Nr. 110 col. 89 und Nr. 125 col. 1007).

${ }^{35}$ Vat. lat. 5567. Nach der Widmung an Karl V. hat Petrus Galatinus den Kommentar „nuperrime“ auf Aufforderung seitens des Kardinals (seit 1527) Francisco Quiñones, tit. S. Crucis, verfaßt (fol. I-II). Quiñones (1475-1540) war zweimal Generalminister der Franziskanerobservanten. Die Vorrede gibt einen Blick auf die Auslegungsgeschichte bis zu Haymo von Auxerre und Albertus Magnus (beider Auslegungen werden als „konfus“ bezeichnet). „At Abbas Joakim ipsum prae / caeteris eleganter et spiritualiter elucidat.“ Weiter werden „Petrus Iohannes“ (Olivi), Ugo Cardinalis (von St. Cher), Albertinus und Lyra genannt (der den Text „per singula verba pulchre ac mirifice clariorem reddit"). Keiner aber „verum ac proprium eius Intellectum ... attingere potuit“. Da die Zeit der endgültigen Enthüllung aller Geheimnisse nun schon nahe bevorstehe, habe Petrus Galatinus sich an die Auslegung gemacht (fol. III ${ }^{\eta}$ ). Vgl. auch Roberto Rusconi, Collezionismo e circolazione di testi profetici tra ' 400 e '500: la figura di Pietro Galatino, in: Continuità e diversità (oben Anm. 3). 
ment war, aus dem die Kirchenrevolution des 16. Jahrhunderts ideologisch auch erwuchs. Ohne die Diagnose des Papstes als des Antichrist durch Luther hätte es keine Reformation gegeben. Die Anwendung der Endzeittexte der Bibel auf die konkrete Gegenwart der Kirche ist ein Element, das sich bei Augustin noch nicht findet, aber im Mittelalter von Bernhard von Clairvaux bis zu Joachim und besonders zu den Spiritualen hoch entwickelt worden ist. Die humanistisch-kirchenreformerische Verbreitung und Abflachung der hier entstandenen Vorstellung von der „babylonischen Gefangenschaft der Kirche" und von einer besseren, erneuerten Kirche liegt der Reformation als ein Ertrag des Mittelalters voraus, als eine neue Waffe, zu der auch gegriffen werden konnte und die sich dann als sehr scharf erwies. 
